\title{
Importance of post-shock streams and sheath region as drivers of intense magnetospheric storms and high-latitude activity
}

\author{
K. E. J. Huttunen ${ }^{1}$ and H. E. J. Koskinen ${ }^{1,2}$ \\ ${ }^{1}$ Department of Physical Sciences, Theoretical Physics Division, P.O. Box 64, FIN-00014 University of Helsinki, Finland \\ ${ }^{2}$ Finnish Meteorological Institute, P.O. Box 503, 00101 Helsinki, Finland
}

Received: 23 June 2003 - Revised: 28 August 2003 - Accepted: 4 September 2003 - Published: 8 April 2004

\begin{abstract}
Magnetic disturbances in the Earth's magnetosphere can be very different depending on the type of solar wind driver. We have determined the solar wind causes for intense magnetic storms $\left(D_{s t}<-100 \mathrm{nT}\right)$ over a 6-year period from the beginning of 1997 to the end of 2002, using observations by the WIND and ACE spacecraft. We have taken into consideration whether the storm was caused by the sheath region or by the following interplanetary coronal mass ejection (ICME). We also divided ICMEs into those having a magnetic cloud structure and those without such a structure. We found that post-shock streams and sheath regions caused the largest fraction of intense magnetic storms. We present four periods of magnetospheric activity in more detail. One of the events was caused by a magnetic cloud (10-11 August 2000) and the rest (13-14 July 2000, 8-9 June 2000 and 17-18 April 2001) by sheath regions and post-shock streams. We have used several magnetic indices to monitor the lowand high-latitude magnetospheric response to these different solar wind structures. Two of the events are interesting examples where at first strong high-latitude activity took place and the low-latitude response followed several hours later. These events demonstrate that low- and high-latitude activity do not always occur concurrently and the level of activity may be very different. According to the examples shown the evolution of the pressure-corrected $D_{s t}$ index was more difficult to model for a sheath region or a post-shock stream driven storm than for a storm caused by a magnetic cloud.
\end{abstract}

\section{Introduction}

One of the main challenges in space weather is to predict the magnetospheric response from the solar wind conditions upstream of the Earth. The main causes of magnetic activity at the Earth are interplanetary manifestations of coronal mass ejections (CMEs), particularly a subset called magnetic

Correspondence to: E. Huttunen

(emilia.huttunen@helsinki.fi) clouds (Burlaga et al., 1981), post-shock streams and fast solar wind streams (Tsurutani et al., 1988; Gosling et al., 1991; Richardson et al., 2001). Magnetospheric activity is often generated by southward interplanetary magnetic fields (IMF) in a region of piled-up solar wind plasma in front of the CME ejecta called the sheath region (Tsurutani et al., 1988). In the sheath region solar wind plasma is heated and compressed, and the draping of the IMF ahead of the CME ejecta can cause intense southward $B_{z}$ events. A shock is a larger scale structure than the CME ejecta driving it. Thus, in many cases spacecraft upstream of the Earth observe only a shock followed by a disturbed solar wind flow (i.e. post-shock stream) but the CME ejecta itself is missed. Practically all interplanetary shocks observed at $1 \mathrm{AU}$ have been demonstrated to be driven by CMEs (Sheeley et al., 1985), whereas the corotating interaction regions develop shocks mainly at larger distances from the Sun. It can make a big difference which type of solar wind structure causes the storm. Magnetic clouds have smoothly changing magnetic field direction and typically low dynamic pressure (a few $\mathrm{nPa}$ ), whereas a rapidly fluctuating magnetic field direction and high dynamic pressure (up to several tens of $\mathrm{nPa}$ ) are characteristic for sheath regions and post-shock streams. These different solar wind conditions put the magnetosphere under very different driving. Huttunen et al. (2002a) showed that sheath regions and post-shock streams generated more $K_{p}$ activity and magnetic clouds enhanced more $D_{s t}$. This suggests that the magnetospheric current systems have different responses during different types of solar wind drivers.

In this paper we examine the drivers of intense magnetic storms $\left(D_{s t}<-100 \mathrm{nT}\right)$ that occurred during the period from 1997 to 2002, i.e. during the rising, maximum and early declining phases of solar cycle 23 . We focus on comparing storms caused by post-shock streams and sheath regions to storms caused by magnetic clouds, because solar wind conditions during these structures are most distinctly different. We investigate four cases in more detail to see the differences in the magnetospheric response driven by different solar wind structures. We have used several magnetic indices, 
described briefly in Sect. 2.1, to monitor the low- and highlatitude magnetospheric response. In Sect. 2.2 we discuss the definition of a magnetic storm based on different activity indices. We have applied two $D_{s t}$ models that are described in Sect. 2.3 to predict the time evolution of $D_{s t}$ for four example events presented in Sect. 3.2. The purpose of this paper is to show that sheath regions and post-shock streams are efficient drivers of intense $D_{s t}$ storms and we demonstrate that, on the other hand, these structures sometimes generate intense and relatively long lasting high-latitude activity though $D_{s t}$ and $S Y M H$ do not decrease concurrently.

\section{Analysis procedure}

\subsection{Description of magnetic indices}

The level of magnetic activity is continuously monitored by various magnetic indices calculated from the measurements of different sets of magnetometer observatories distributed around the Earth (Mayaud, 1980). The $D_{s t}$ index is calculated every hour from perturbations of the horizontal component $(H)$ of the geomagnetic field using measurements of four low-latitude magnetometer stations. Physically, $D_{s t}$ aims to measure the strength of the symmetrical equatorial ring current, but it is contaminated by other current systems (e.g. magnetopause current, tail current, field-aligned currents). In this study final values of $D_{s t}$ were available for 1997-2001 and provisional values were used for the year 2002.

The SYMH index (Iyemori, 1990; Iyemori and Rao, 1996) is essentially the same as the $D_{s t}$ index, but has a higher time resolution (1 $\mathrm{min}$ ) and is calculated from a partly different set of six stations. $S Y M H$ is obtained by averaging the disturbance component at each minute for the selected 6 stations. $A S Y H$ is an asymmetric disturbance component in the $S Y M H$ index. The longitudinally symmetric component is subtracted from the disturbance field for each station. $A S Y H$ is obtained as the range between the maximum and minimum deviations. $D_{s t}, S Y M H$ and $A S Y H$ monitor mainly low-latitude activity (i.e. evolution of the ring current), although 1-min $S Y M H$ and $A S Y H$ are more sensitive to the substorm related activity (e.g. tail current disruption at the substorm onset).

The $A E$ index is derived as 1-min values from geomagnetic variations in the $H$ component using a chain of 10-13 magnetometer stations distributed under the Northern Hemisphere auroral zone. AE measures the strength of the auroral electrojets and thus represents the substorm activity well. When the overall level of magnetic activity is high, the confidence of $A E$ is reduced as the auroral oval moves equatoward. However, for all presented events in Figs. 2-5, AE should give a good estimate during the whole storm period.

$K_{p}$ is a range index obtained from 13 subauroral stations. First, the local disturbance levels at each stations are determined by taking the largest excursion in the horizontal components during a 3-h time interval. The range is con- verted into a local $K$-index using the station specific quasilogarithmic scale. The effects of annual and daily variations are eliminated by applying conversion tables. This determines the standardized $K$ index, $K_{s}$, for each station that is expressed in the range 0-9 in steps of one unit. A 3-h global geomagnetic index, $K_{p}$, is obtained as an average of $K_{s}$ values. Variations in $K_{p}$ are difficult to interpret physically since depending on the level of magnetic activity, the $K_{p}$ stations are under the influence of different magnetospheric current systems. Thus, $K_{p}$ is more sensitive to high-latitude activity than $D_{s t}$. All magnetic indices described above are available at the World Data Center for Geomagnetism, Kyoto.

\subsection{Definition of a storm}

We briefly discuss the definition of a magnetic storm in the context of the indices used in this study. Generally, a magnetic storm is understood as an increase in particle energy densities encircling in the equatorial ring current, which causes a global depression of the low-latitude $\mathrm{H}$ component of the Earth's geomagnetic field lasting several hours (e.g. Kamide et al., 1997). The strength of a magnetic storm is usually defined by the minimum $D_{s t}$. The $D_{s t}$ value $-50 \mathrm{nT}$ is often used as a threshold for a moderate storm, and $-100 \mathrm{nT}$ as a threshold for an intense storm (e.g. Gonzalez et al., 1994). However, it has become clear that $D_{s t}$ measures more than just the symmetric ring current. It has been documented both observationally and in theoretical models (e.g. Greenspan and Hamilton, 2000; Liemohn et al., 2001) that in the storm main and early recovery phases, the ring current is highly asymmetric. Up to $90 \%$ of the magnetic field depression during a storm can be due to ions in open trajectories (Liemohn et al., 2001), i.e. they drift around the Earth on the dusk side and disappear on the dayside magnetosphere. Also, other current systems, especially the tail current, may have a significant contribution to $D_{s t}$ (Ganushkina et al., 2003; Turner et al., 2000; McPherron, 1997). Thus, it should be pointed out that when we talk about the ability of different solar wind structures to generate intense $D_{s t}$ storms, it is not exactly consistent with their ability to increase the amount and energy of particles encircling in a ring current. The relative contribution from different current systems may be different in storms caused by different solar wind structures.

The $K_{p}$ index is also widely used as a storm indicator, though it is more difficult to interpret physically and may have a significant contribution from high-latitude auroral currents. According to Tsurutani et al. (1997), magnetic activity is defined as moderate if $K_{p}$ is 5 or larger, and intense if $K_{p}$ exceeds 6. Gosling et al. (1991) and Richardson et al. (2001) defined a large storm to have $7-\leq K_{p_{\max }} \leq 7+$ and $K_{p} \geq 6-$ for at least three 3-h intervals during a 24 -h period. In this work we investigate both low- and high-latitude magnetic activity. In Sect. 3 we show two examples where a sheath region generated high level $A E$ and $K_{p}$ activity, but $D_{s t}$ and $S Y M H$ indices were not depressed to storm-time values at the same time. 


\section{$2.3 D_{s t}$ models}

To predict the evolution of $D_{s t}$ using solar wind parameters as input we have used two modelling schemes developed by O'Brien and McPherron (2000a) (M1) and by Fenrich and Luhmann (1998) (M2). Both of them have a clear physical content and they are easy to use. The M1 model is adjusted to storms with $D_{s t}>-150 \mathrm{nT}$ and have been proved to establish well the $D_{s t}$ changes during several storm periods (O'Brien and McPherron, 2000b). The pressure-corrected $D_{s t}\left(D_{s t}^{*}\right)$ is calculated by removing the effect of the magnetopause Chapman-Ferraro currents using the formula by Burton et al. (1975):

$D_{s t}^{*}=D_{s t}-b \sqrt{P_{\mathrm{dyn}}}+c$.

For constants $a$ and $b$, we have used values $b=7.26 \mathrm{ntT}(\mathrm{nPa})^{1 / 2}$ and $c=11 \mathrm{nT}$ derived by O'Brien and McPherron (2000a). The evolution of $D_{s t}^{*}$ is covered by the Burton et al. (1975) formula:

$\frac{d\left(D_{s t}^{*}\right)}{d t}=Q(E)-\frac{D_{s t}^{*}}{\tau}$

where $Q(E)$ is a ring current injection function and $\tau$ is a ring current decay parameter. For $Q(E)$ the M1 model uses a linear function of solar wind $E_{y}=V B_{S}$ similar to Burton et al. (1975) with slightly modified coefficients:

$$
Q(E)= \begin{cases}0 & E_{c}<0.49 \mathrm{mV} / \mathrm{m} \\ -4.4 \times\left(E_{y}-E_{c}\right) & E_{c}>0.49 \mathrm{mV} / \mathrm{m} .\end{cases}
$$

The injection term in the M2 model depends on the solar wind dynamic pressure:

$Q(E)=-4.3\left(P_{\mathrm{dyn}}\right)^{1 / 3}\left(E_{y}-0.5\right)$.

The coefficient -4.3 is chosen so that $Q(E)$ is consistent with the Burton et al. (1975) value when $P_{\mathrm{dyn}}$ is $2 \mathrm{nPa}$. Thus, a $P_{\text {dyn }}$ larger than $2 \mathrm{nPa}$ increases the ring current injection. For the M1 model the ring current decay parameter $(\tau)$ varies as a function of $V B_{S}$ :

$\tau($ hours $)=2.4 e^{9.74 /\left(4.69+V B_{S}\right)}$.

In the M2 model $\tau$ has a constant value $7.7 \mathrm{~h}$ when $E_{y}$ is less than $4 \mathrm{mV} / \mathrm{m}$ and 3 , or $5 \mathrm{~h}$ (the value that gives the best fit is chosen) is used otherwise.

\section{Events}

\subsection{Drivers of intense magnetospheric storms}

We investigated the solar wind causes of intense $\left(D_{s t}<-100 \mathrm{nT}\right)$ magnetic storms during the 6-year interval (1997-2002). In total, 56 intense magnetic storms occurred and in 53 cases we could identify the solar wind driver. We decided not to use the pressure corrected $D_{s t}$ as it is not statistically important whether the pressure corrected

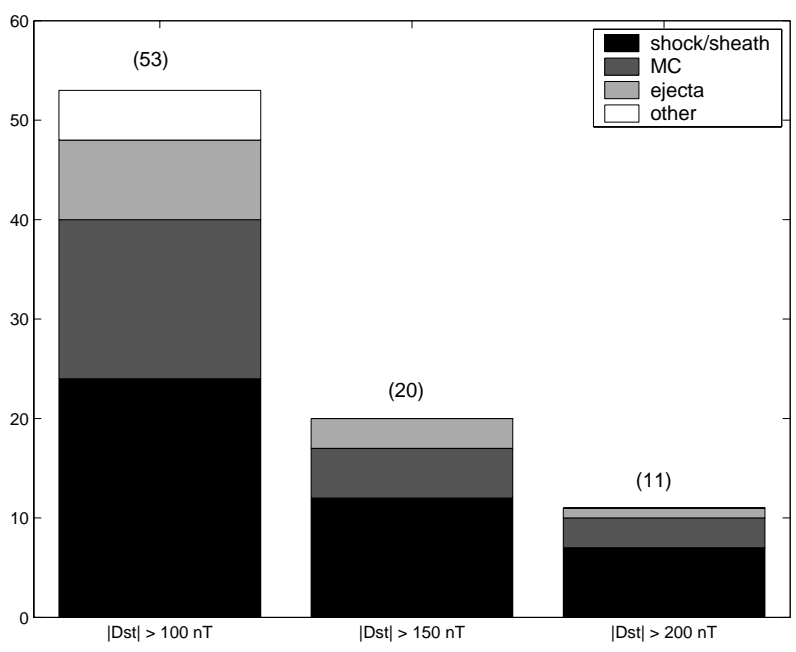

Fig. 1. Bars show the drivers of magnetic storms for three different level of the $D_{s t}$ depression. The numbers above each bar present the total number of storms in that category.

values are used or not for the peak $D_{s t}$. If the pressure corrected values would have been used, the importance of post-shock streams and sheath regions as storm drivers would have slightly increased, as these structures are associated with high dynamic pressure and thus have a large pressure correction. A solar wind driver of a storm was defined as the feature during which the $D_{s t}$ index reached $85 \%$ of its minimum for this particular storm. Figure 1 shows the drivers of magnetic storms for three different limits of the maximum $\left|D_{s t}\right|: 100 \mathrm{nT}, 150 \mathrm{nT}$, and $200 \mathrm{nT}$. We classified the drivers of storms in the following categories: post-shock stream or sheath region (shock/sheath), magnetic cloud (MC), CME ejecta without the magnetic cloud structure (ejecta) and solar wind causes not associated with shocks or CME ejecta (other). We identified solar wind drivers from ACE or WIND magnetic field and solar wind measurements. Magnetic clouds were identified using the definition by Burlaga et al. (1981). Note that the categories "MC" and "ejecta" also include a few cases where sheath region fields preceding the magnetic cloud contributed significantly (but less than $85 \%$ of the $D_{s t}$ minimum) to the $D_{s t}$ depression.

The first bar in Fig. 1 shows the storms for which the maximum $\left|D_{s t}\right|$ was $>100$ nT. Sixteen of 53 storms (30\%) had a main contribution from southward magnetic cloud fields. The largest fraction of storms, 24 (45\%, was caused by a post-shock stream or by a sheath region. In the next bar the limit of $\left|D_{s t}\right|$ has been increased to $150 \mathrm{nT}$, and the number of magnetic storms is reduced to 19 . The relative contributions from shocks and sheath regions is now larger; they caused $12(60 \%)$ storms, whereas the relative contribution of magnetic clouds decreased. Magnetic clouds caused only 5 storms (20\%). And finally, the last panel shows drivers for 11 magnetic storms that had the maximum $\left|D_{s t}\right|>200$ nT. Postshock streams or sheath regions caused 7 of these storms, and magnetic clouds caused only 2 or 3 storms. The driver of the biggest storm of solar cycle 23 (31 March 2001) is unclear. 
Four of the storms caused by sheath region fields were preceded by a sequence of multiple fast CMEs observed by the LASCO instrument on board SOHO. The sheath region for these cases probably contained piled-up material from the preceding $\mathrm{CME}(\mathrm{s})$. The more detailed study of these major magnetic storms $\left(D_{s t}<-200 \mathrm{nT}\right)$ is given in Huttunen et al. (2002b). The solar wind driver and magnetospheric consequences of the strong storm on 6-7 April 2000 are described in detail in Huttunen et al. (2002c).

\subsection{Events}

Four periods of solar wind data and magnetospheric indices are presented in Figs. 2-5. Solar wind data is obtained from the ACE satellite located near the L1 point, more than $200 R_{E}$ from the Earth. All solar wind data have been shifted from ACE to the magnetopause. The data before the shock have been shifted by the approximate downstream solar wind speed and the data after the shock by the approximate upstream solar wind speed. The overlapping data points were omitted as physically they were hidden inside the shock structure. Figures $2-5$ are in the same format, showing from top to bottom: solar wind $B_{z}$ component in the GSM coordinate system (a) and solar wind speed (b). Together with the solar wind dynamic pressure (blue line) (c) the subsolar magnetopause location (green line) is given from the empirical model by Shue et al. (1998) that estimates the magnetopause location as a function of IMF $B_{Z}$ and the solar wind dynamic pressure. The dashed line marks the geostationary orbit. The energy input to the magnetosphere is estimated by the epsilon parameter $\left(\varepsilon=10^{7} V B^{2} l_{o}^{2} \sin ^{4}(\theta / 2)\right.$, using SI-units and $\left.l_{o}=7 R_{E}\right)(\mathrm{d})$. The last four panels show magnetic indices: $D_{s t}$ (solid black line), pressure corrected $D_{s t}$ (dashed blue line) and the modelled $D_{s t}^{*}$ indices: The prediction from the M1 model is shown by open red circles and from the M2 model by filled green circles (e); SYMH (blue dashed line), pressure corrected $S Y M H$ (red dashed line) and negative $A S Y H$ (black solid line) (f); $K_{p}$ (g) and finally, $A E$ and $A L$ indices (h).

\subsubsection{0-11 August 2000 (AUG2000)}

Figure 2 shows an example of an intense magnetic storm driven by a slow speed magnetic cloud that was observed by ACE on 10-11 August 2000. There was a small shock at ACE at 04:07 UT on 10 August. The magnetic cloud started at $\sim$ 19:00 UT on 10 August, identified from a drop in plasma beta (not shown) and the start of slow the magnetic field change. The $B_{z}$ minimum was $-14 \mathrm{nT}$ at the leading edge, from which the southward component rather smoothly decreased during the passage of the cloud. Dynamic pressure was only a few $\mathrm{nPa}$. As a consequence, the subsolar magnetopause was not significantly compressed from the quiet-time position $\left(\sim 11 R_{E}\right)$.

The energy injection to the magnetosphere was steady, $\sim 1.7 \times 10^{12} \mathrm{~W}$ for about $5 \mathrm{~h}$ and afterwards began to weaken slowly. $D_{s t}^{*}$ started to decrease after the magnetic cloud ar- rival and reached the minimum of $-102 \mathrm{nT}\left(D_{s t}:-106 \mathrm{nT}\right)$ on 11 August 2000 at 07:00 UT. From Fig. $2 \mathrm{f}$ it is seen that the symmetric part of $H$ was larger than the asymmetric part during the whole storm period. $K_{p}$ had storm-time values ( $\geq 5$ ) for $12 \mathrm{~h}$ with a maximum value of 6 on 11 August, 00:00-06:00 UT. Thus, this was not a large storm according to the Gosling et al. (1991) $K_{p}$-criteria. The $A E$ index shows continuous substorm activity. The $A L$ index did not recover near the zero level, so the number of substorms is hard to determine. Both the M1 model and the M2 model (with $\tau=5$ ) give good estimates of the $D_{s t}^{*}$ behaviour and the minimum value. The M2 model gives a better prediction for the recovery phase than M1.

\subsubsection{7-18 April 2001 (APR2001)}

Figure 3 shows another intense magnetic storm that was caused by a disturbed solar wind flow after a strong shock observed at ACE on 18 April at 00:05 UT. The $D_{s t}$ minimum was about the same as for AUG2000. The comparison of this event to AUG2000 reveals many differences in the characteristics of the solar wind driver and the storm evolution. At the shock the solar wind speed rose from about $380 \mathrm{~km} / \mathrm{s}$ to $500 \mathrm{~km} / \mathrm{s}$, and solar wind temperature, density, and the magnetic field magnitude had clear jumps. During several hours after the shock, the IMF had irregular behaviour, fluctuating from the south to the north. At the shock the dynamic pressure increased from a few $\mathrm{nPa}$ above $10 \mathrm{nPa}$ and remained high for several hours. Figure $3 \mathrm{c}$ shows that the dayside magnetopause was strongly compressed during a 6-h period (i.e. during high dynamic pressure), most of that time beyond the geostationary orbit.

There was one longer interval of southward IMF from about 02:00 to 04:00 UT on 18 April, with a minimum value of $-25 \mathrm{nT}$. The energy input was strong, up to $6 \times 10^{12} \mathrm{~W}$, but irregular. The main phase of this storm lasted only $5 \mathrm{~h}$. $D_{s t}^{*}$ reached the minimum value $-128 \mathrm{nT}\left(D_{s t}:-114 \mathrm{nT}\right)$ on 18 April at 07:00 UT. Figure $2 \mathrm{f}$ shows that during the storm main phase the symmetric part of $H$ was clearly larger than the asymmetric part. The high-latitude response was stronger than during AUG2000. $K_{p}$ had 9-h period storm-time values $(6,7+, 7-)$, with the maximum being 7+ on 18 April, 03:0006:00 UT. Note that during this 9-h period all $K_{p}$ values were larger than the maximum $K_{p}$ for AUG2000. The level of the $A E$ activity was high, reaching $1800 \mathrm{nT}$. $A L$ shows that four substorms occurred during the main phase. The most intense one $(\sim-1500 \mathrm{nT})$ took place before the start of the $D_{s t}$ and the $S Y M H$ decrease. For this event the M1 model gives a rather good estimation of the $D_{s t}$.

\subsubsection{3-14 July 2000 (JUL2000)}

Last two events were not intense magnetic storms if determined by $D_{s t}$, but they were associated with strong highlatitude activity. The first of these events is shown in Fig. 4. A strong shock was observed at ACE on 13 July 2000 at 09:18 UT. Note that this event took place 2 days before the 

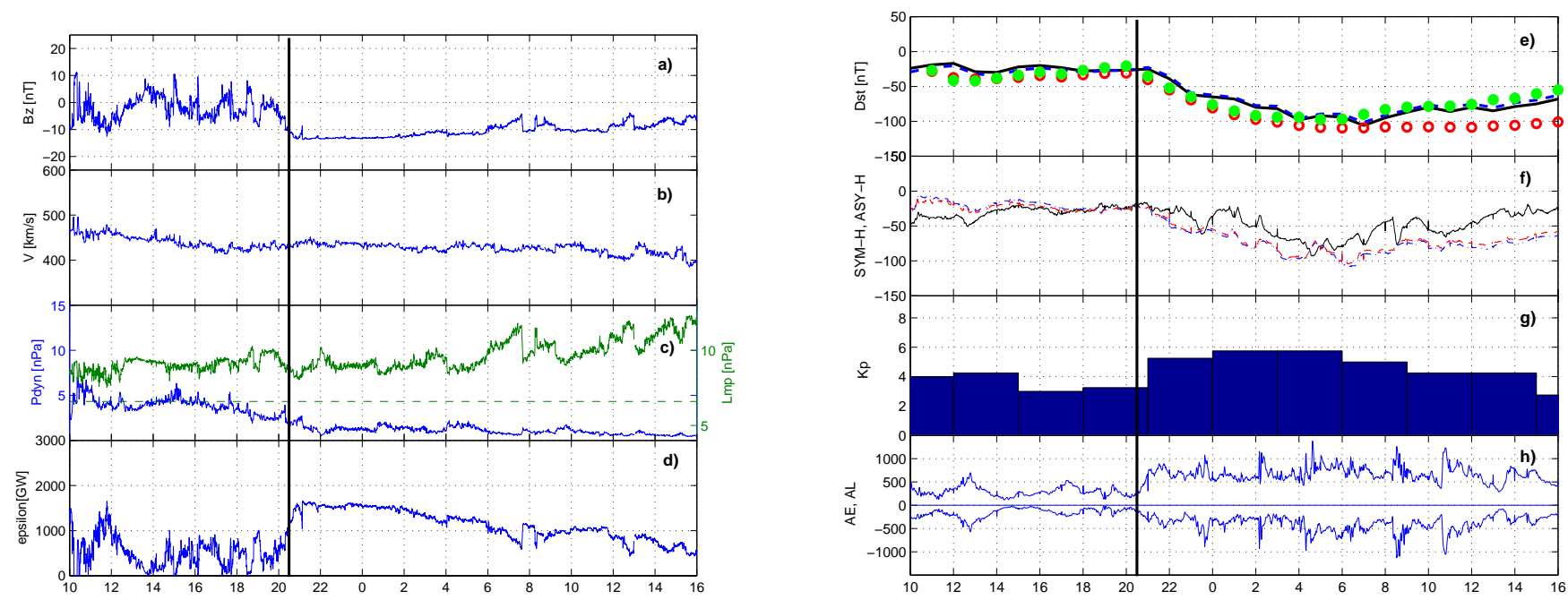

Fig. 2. Solar wind parameters and geomagnetic indices for a 30-h interval from 10 August, 10:00 UT-11 August, 16:00 UT, 2000 measured by ACE. The panels from top to bottom show: IMF $B_{z}$ component (a), solar wind speed (b), dynamic pressure (blue line) with the estimated subsolar magnetopause position from the Shue et al. (1998) model (green line). The vertical dashed line indicates the geosynchronous orbit (c), the $\varepsilon$-parameter (d), the $D_{s t}^{*}$ index (black solid line) and the modelled $D_{s t}^{*}$ indices: Open red circles give the prediction by O'Brien and McPherron (2000a) and filled red circles by Fenrich and Luhmann (1998) (e), the $S Y M H$ index (blue line), the pressure corrected $S Y M H$ (red line) and the $A S Y H$ index (black line) (f), $K_{p}(\mathbf{g})$ and $A E$ and $A L$ indices (h). The solid line indicates the start time of the magnetic cloud.
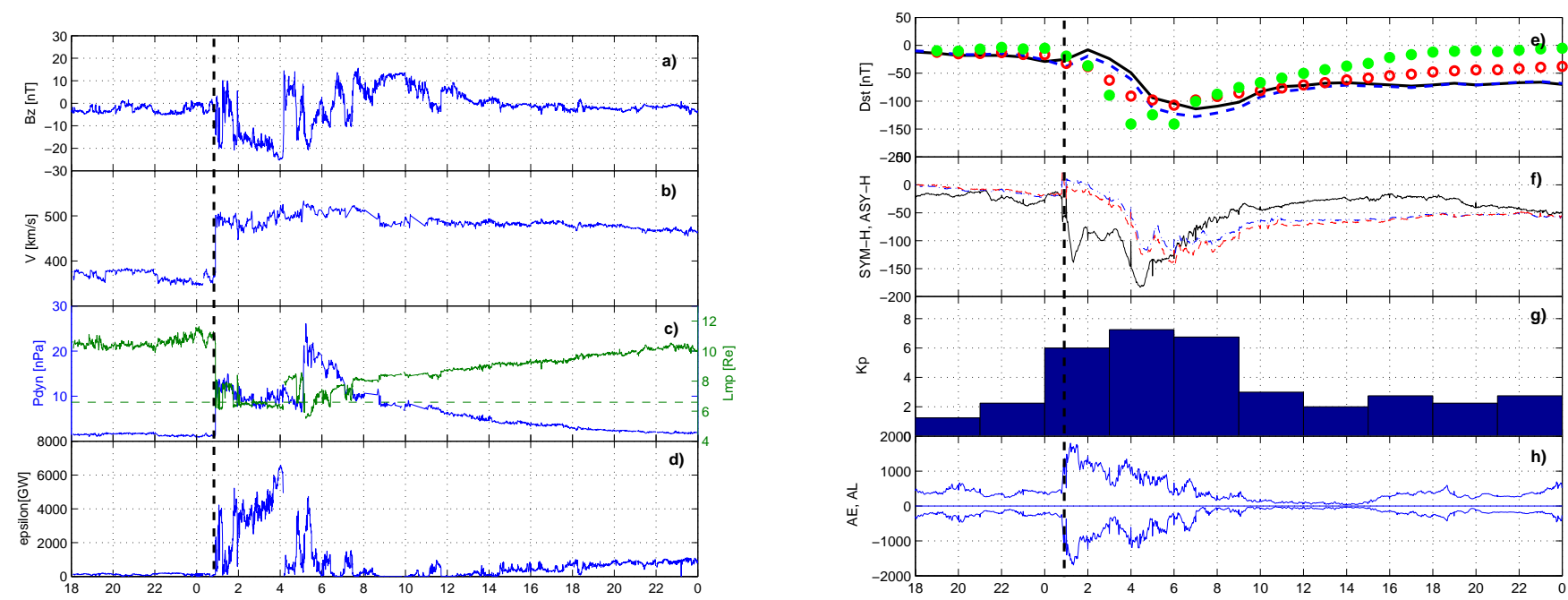

Fig. 3. Solar wind parameters and geomagnetic indices for a 30-h interval from 17 April, 18:00 UT-18 April, 24:00 UT, 2001 measured by ACE. The panels from top to bottom are the same as in Fig. 2. The dashed line indicates the shock.

famous Bastille Day CME hit the magnetosphere (Lepping et al., 2001). After the shock the magnetic field direction fluctuated rapidly from the south to the north, with the minimum $B_{z}$ about $-20 \mathrm{nT}$. At the shock dynamic pressure increased above $20 \mathrm{nPa}$ and remained high until about 17:00 UT on 13 June. From that time to about 02:00 UT on 14 June the magnetic field had regular behaviour and the solar wind dynamic pressure decreased to a few $\mathrm{nPa}$, suggesting that ACE observed a CME ejecta. The subsolar magnetopause was significantly compressed and fluctuated around the geostationary orbit from 10:00 to 15:00 UT.
The $\varepsilon$-parameter shows that the energy injection was very sporadic but strong, with peaks reaching $6 \times 10^{12} \mathrm{~W}$ (i.e. values comparable with APR2001). There was also some energy input between 19:00-23:00 UT due to southward CME ejecta fields, but the magnitude of the southward component was only $\leq 4 \mathrm{nT}$ and the $\varepsilon$-parameter was $5-9 \times 10^{11} \mathrm{~W}$. During the sheath region passage, high-latitude activity was strong. $K_{p}$ had storm-time values $(6-, 7,6+)$ for a 9-h period (09:00-18:00 UT) and at the same time $A E$ was enhanced up to $\sim 1100 \mathrm{nT}$. In total, were 6 substorms, but most of them were only a few hundred nT. The most intense one was about 

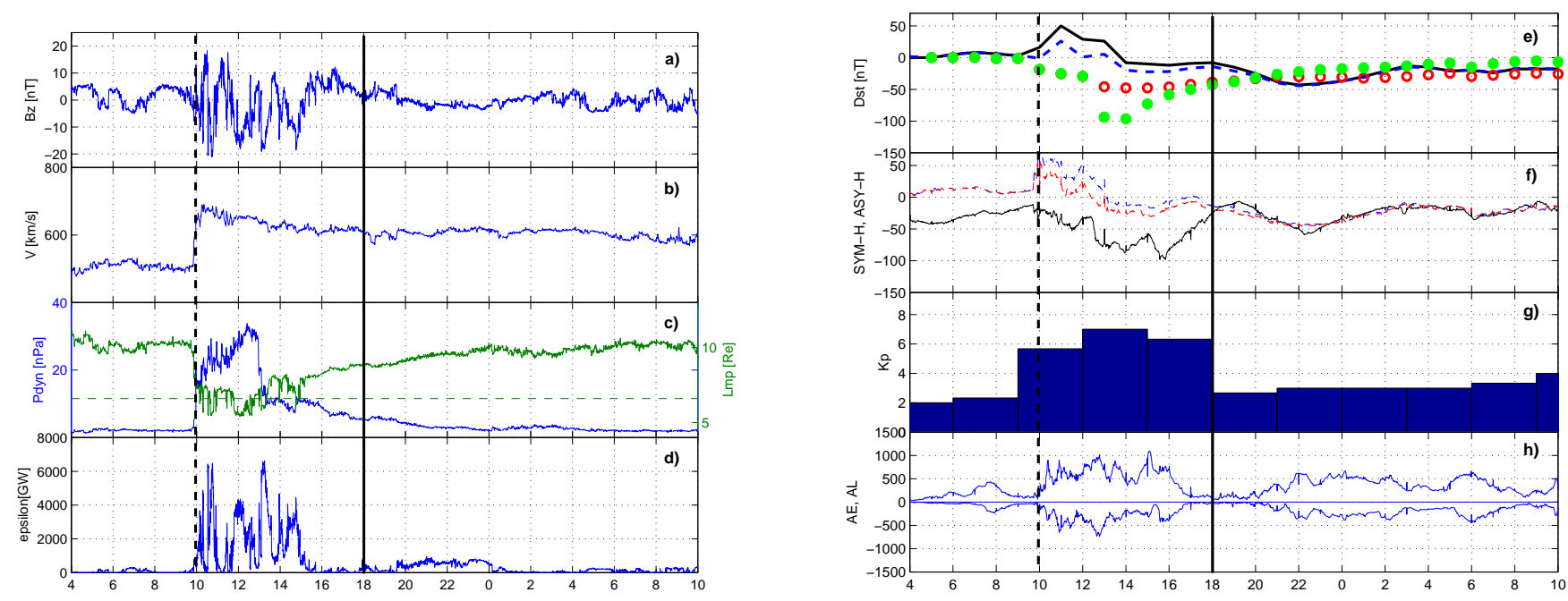

Fig. 4. Solar wind parameters and geomagnetic indices for a 30-h interval from 13 July, 04:00 UT-14 July, 10:00 UT, 2000 measured by ACE. The panels from top to bottom are the same as in Fig. 2. The dashed line indicates the shock and the solid line the arrival of the CME.

$-700 \mathrm{nT}$. During the period of the strong high-latitude activity, $D_{s t}^{*}$ and $S Y M H$ stayed around the quiet level. Large positive $D_{s t}^{*}$ values were observed during the pressure pulse and most likely, the pressure correction (Eq. 1) used was not enough to remove the effect of the magnetopause currents. Several hours later, when high-latitude activity had already weakened, $D_{s t}^{*}$ decreased to $-45 \mathrm{nt}$ at 20:02 UT on 13 June. Despite the weak $D_{s t}$ response this event fulfilled the $K_{p^{-}}$ criteria by Gosling et al. (1991) for a large storm . Both models, especially the M2 model (with $\tau=3 \mathrm{~h}$ ), predict a $D_{s t}$ depression during the sheath passage that is too large and when the measured $D_{s} t^{*}$ decreased, the models predict the recovery of $D_{s t}^{*}$.

On the next day, 14 July $2000, K_{p}$ again had high values (5-7+7-) for a 9-h interval and the $A E$ activity reached almost $2000 \mathrm{nT}$ with no concurrent decrease in $D_{s t}$ or $S Y M H$. This event was caused by a fluctuating magnetic field following a shock seen by ACE on 14 July at 14:59 UT. $D_{s t}$ started to decrease on 14 July at 21:00 UT and reached the minimum value, $-34 \mathrm{nT}$, on 15 July at 01:00 UT. We could not use this event for further study, as both WIND and ACE lacked solar wind measurements. However, magnetic field data show that from 21:00 UT on 14 July when $D_{s t}$ started to decrease to the time of the $D_{s t}$ minimum at 01:00 UT on $15 \mathrm{July}, B_{z}$ (in GSM) had slightly northward values (2-6nT). Thus, the $D_{s t}$ models used would likely have failed to predict the $D_{s t}$ depression similar to the event shown in Fig. 4.

\subsubsection{8-9 June 2000 (JUN2000)}

The last event is shown in Fig. 5. ACE detected a strong shock on 8 June 2000 at $08: 41$ UT. For about $8 \mathrm{~h}$ after the shock the magnetic field direction fluctuated and the solar wind dynamic pressure, was high. On 8 June starting at about 16:00 UT, ACE observed regular magnetic field behaviour and decreasing dynamic pressure indicating the presence of a
CME ejecta. During the sheath region ( 09:00-16:00 UT), the magnetopause was compressed beyond the geostationary orbit. The $\varepsilon$-parameter shows again strong but irregular energy input. The $A E$ and $K_{p}$ indices were most strongly enhanced during the sheath region passage. The $A E$ maximum was about $1900 \mathrm{nT}$ and before the start of the $D_{s t}$ and $S Y M H$ decrease three substorms took place. During the main phase there were two substorms. $K_{p}$ had storm-time values of $15 \mathrm{~h}$ and a maximum value of 7, 12:00-15:00 UT on 8 June. $D_{s t}^{*}$ started to decrease after 16:00 UT about the time of the arrival of the CME ejecta. The $D_{s t}^{*}$ minimum was $-90 \mathrm{nT}$ on 8 June at 20:00 UT. The M1 model predicts somewhat lower $D_{s t}^{*}$ than the measured during the strong highlatitude activity. When the measured $D_{s t}^{*}$ decreased by almost $100 \mathrm{nT}$ to its minimum value the model predicts only about a $30 \mathrm{nT}$ decrease. The M2 model (with $\tau=3 \mathrm{~h}$ ) predicts correctly the depth of the $D_{s t}^{*}$ minimum, but too early.

\section{Discussion}

During the six year period (1997-2002, 53 intense $\left(D_{s t}<-100 \mathrm{nT}\right)$ magnetic storms occurred for which we could identify the solar wind cause. We found that postshock streams and sheath regions were the most important storm drivers, causing nearly half (45\%) of the storms. The importance of post-shock streams and sheath regions was increased when the limit of the $D_{s t}$ depression was increased. Tsurutani et al. (1988) showed, based on 10 magnetic storms that sheath regions alone can drive intense magnetic storms. Note that we defined the cause of a storm as the solar wind structure that led to $85 \%$ of the $D_{s t}$ minimum of that particular storm. Defining the cause as the structure that led $D_{s t}$ to cross our storm limits $(-100,-150$ or $-200 \mathrm{nT}$, see Fig. 1) would have increased the importance of shocks and sheath regions as storm drivers. The period of our study 

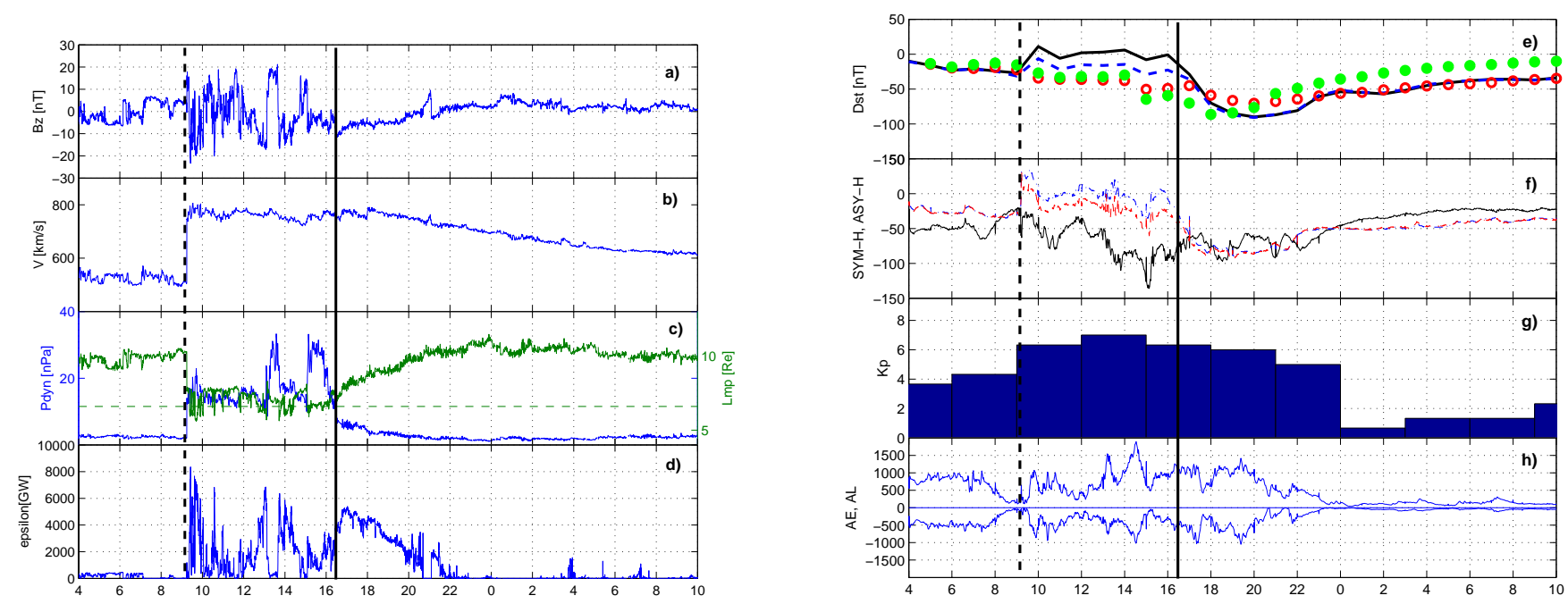

Fig. 5. Solar wind parameters and geomagnetic indices for a 30-h interval from 8 June, 04:00 UT-9 June, 10:00 UT, 2000 measured by ACE. The panels from top to bottom are the same as in Fig. 2. The dashed and solid lines are the same as in Fig. 4.

covers rising, maximum and early declining phases of solar cycle 23. Thus, during most of the period the CME activity was high. According to the WIND magnetic cloud list at http://lepmfi.gsfc.nasa.gov/mfi/mag_cloud_pub1.html 60 magnetic clouds were identified during the years 19972002. This means that about one-fourth of magnetic clouds that hit the Earth caused an intense magnetic storm. The total number of shocks observed at $1 \mathrm{AU}$ during the period of our study was much larger than the number of magnetic clouds. According to the ACE shock list at http:// www.bartol.udel.edu/ $\sim$ chuck/ace/ACElists/obs_list.html, approximately 200 shocks (only clear cases included) were observed during 1997-2002. Thus, about one-tenth of postshock streams and sheath regions caused an intense magnetic storm. Although southward sheath region fields led to the $D_{s t}$ minimum in such a large fraction of intense storms, the following magnetic cloud having southward fields are important in the sense that $D_{s t}$ recovers slowly, i.e. magnetic cloud fields do not necessarily cause further enhancement of $D_{s t}$ but can keep $D_{s t}$ low for a long time. Besides, storms caused by magnetic cloud fields usually have more gradual $D_{s t}$ decrease (i.e. longer main phases) than storms caused by post-shock streams and sheath regions.

Let us consider characteristics and differences of magnetic cloud and sheath region/post-shock stream driven magnetic storms. In Fig. 2 (AUG2000) we presented an intense magnetic storm driven by a magnetic cloud. Typical for magnetic clouds are small variations in solar wind parameters, for example, smoothly changing IMF $B_{z}$ and low dynamic pressure. For the comparison in Fig. 3 we showed an intense magnetic storm driven by a post-shock stream (APR2001). Events JUL2000 and JUN2000 presented in Figs. 4 and 5 were caused by sheath region fields, but had a weaker $D_{s t}$ response. Sheath regions and post-shock streams are associated with irregular behaviour of solar wind variables: the magnetic field direction is highly variable and the dynamic pressure is high with large variations in its magnitude. The draping of magnetic field lines and the compression of solar wind plasma ahead of a CME ejecta lead to large IMF magnitudes and intense southward $B_{z}$ events.

Because of low dynamic pressure the subsolar magnetopause is not significantly compressed during the magnetic cloud passage. A passage of a sheath region or a postshock stream causes significant magnetopause compression, as seen from Figs. 3c-5c. The subsolar magnetopause was compressed several hours beyond the geostationary orbit, and there were large-scale fluctuations in its position. When the magnetopause is strongly compressed the losses of particles drifting to the dayside magnetosphere are enhanced, which in turn may partly keep $D_{s t}$ from developing (Kozyra et al., 2002). On the other hand high dynamic pressure may enhance the ring current injection (Fenrich and Luhmann, 1998).

The energy input to the magnetosphere is steady and continues for a long time during magnetic clouds because of the smooth rotation of the magnetic field direction. Thus, magnetic clouds cause periods of steady convection. On the contrary, during the passage of sheath regions and post-shock streams the energy input is usually irregular, but intense. The larger the convection electric field the closer is the separatrix between the open and closed drift trajectories to the Earth (e.g. Kamide et al., 1997). In our study the peak value of the southward $B_{z}$ during the solar wind driver for intense storms was larger for post-shock streams and sheath regions than for magnetic clouds ( $29 \mathrm{nT}$ and $21 \mathrm{nT}$ with standard deviations $13 \mathrm{nT}$ and $12 \mathrm{nT}$ ). Thus, generally during post-shock streams and sheath regions particles on open trajectories drift closer to the Earth than during magnetic clouds. The effects of the fluctuating convection electric field are twofold: on the one hand, although the energy input is strong, if the periods of enhanced convection are very short in duration (like for JUL2000 and JUN2000), particles are not transported to 
the ring current region. Such a large fraction of post-shock streams and sheath regions cannot drive intense $D_{s t}$, storms since the magnetic field direction fluctuates too rapidly. On the other hand, when the duration of the injection peaks are longer (like for APR2001), post-shock streams and sheath regions seem to efficiently drive intense $D_{s t}$ storms. The weakening of the convection electric field is necessary for trapping particles from open trajectories to closed trajectories (e.g. Gonzalez et al., 1994; Kamide et al., 1997). Kamide et al. (1997) discussed that fluctuations in convection can enhance the ring current increase compared to the steady convection of the same average magnitude when the storm length exceeds $3 \mathrm{~h}$.

It has been demonstrated (Kamide et al., 1997; Kozyra et al., 2002) that the nightside plasma sheet source population changes have a great importance in the ring current evolution. Presumably, strong high-latitude activity and solar wind pressure pulses during the sheath region or post-shock stream passage cause an enhanced outflow of $\mathrm{O}^{+}$ions, as both the high $A E$ activity and high solar wind dynamic pressure are shown to correlate with $\mathrm{O}^{+}$energy density in the near-Earth magnetotail (Daglis et al., 1994). Furthermore, high densities typical for sheath regions and post-shock streams may lead to a "super-dense" plasma sheet, that if swept from nightside to dayside, will lead to an extra decrease in $D_{s t}$ (Borovsky et al., 1997). Thus, sheath regions and post-shock streams provide a rich particle source population in the near-Earth plasma sheet. When these particles are injected into the inner magnetosphere, $D_{s t}$ decreases. During the magnetic cloud passage, the plasma sheet particle population decreases and although the convection remains high, $D_{s t}$ recovers during the magnetic cloud because less particles from the plasma sheet are injected into the inner magnetosphere. For example, sheath region fields caused the intense magnetic storm on 6-7 November 2000, although the following magnetic cloud had southward fields at the leading edge and more intense southward IMF values than the sheath region. Liemohn et al. (2001) presented similar events simulating the near-Earth ion distribution with a kinetic transport model.

The evolution of magnetic indices for intense storms AUG2000 and APR2001 was very different, which reflects differences in the response of magnetospheric current systems. For AUG2000 $D_{s t}$ decreased gradually, in $10 \mathrm{~h}$, to the minimum value, whereas for APR2001 the main phase lasted only $5 \mathrm{~h}$. For AUG2000 the symmetric part of $H$ was larger than the asymmetric part of $H$ during the whole storm period. During the main phase of the APR2001 storm, the asymmetric part of $H$ dominated the symmetric part and $A S Y H$ had large variations. Also, for the JUL2000 and JUN2000 events $A S Y H$ had large values during high-latitude activity. For all events peaks in $A S Y H$ were associated with high $A E$ activity. We suggest that a substorm related partial ring current flowing around the dusk side of the Earth caused these ASY H peaks. Iyemori (1990) discussed the morphology of such a current system as inferred from the mid-latitude asymmetric fields. Partly, large $A S Y$ values for sheath regions and post-shock streams can result from periods of intense convec- tion when particles drift close to the Earth and disappear on the dayside magnetopause. A further interpretation would require a closer investigation of the magnetic recordings from magnetometer stations that have been used to derive $A S Y H$ and $S Y M H$ indices for each event. APR2001 had higher level $K_{p}$ and $A E$ activity, while $D_{s t}$ minima were about the same. $K_{p}$ values for APR2001 were larger than the maximum $K_{p}$ for AUG2000. During the passage of a magnetic cloud for AUG2000 the $A E$ activity was continuous. The local IMAGE magnetometers in the midnight sector indicated very complicated current structures (data not shown). Our recent studies (Huttunen et al., 2002c; Pulkkinen et al., 2002) have shown that the auroral activity during magnetic storms can be very variable, sometimes exhibiting clear substormlike processes and sometimes not.

Events JUL2000 and JUN2000 were associated with strong high-latitude activity, comparable to the APR2001 intense storm. For both events $K_{p}$ and $A E$ were strongly enhanced without concurrent decrease in $S Y M H$ or $D_{s t}$. The most intense high-latitude activity had weakened when $D_{s t}$ and $S Y M H$ started to decrease. For the JUL2000 event $A E$ reached $1100 \mathrm{nT}$ and for the JUN2000 event, almost $2000 \mathrm{nT}$. Both events were large storms according to the $K_{p}$ criteria by Gosling et al. (1991), but JUL2000 was only a minor magnetic storm if determined by $D_{s t}$.

For AUG2000 both the M1 and M2 models predicted well the $D_{s t}^{*}$ behaviour and the minimum value of $D_{s t}^{*}$. The problems of the M1 and M2 models in predicting $D_{s t}^{*}$ variations for JUL2000 and JUN2000 are probably due to large and rapid variation of the magnetic field direction. It seems that these high frequencies are filtered out in the magnetosphere. The pressure correction of $D_{s t}$ given by Eq. (1) may not be adequate to remove the effect of the magnetopause current systems. Furthermore, the sheath regions and post-shock streams may cause strong preconditioning of the nightside plasma sheet. The injection of these particles to the ring current region can cause the unpredicted intensification of $D_{s t}^{*}$.

\section{Conclusions}

In this study we have shown that post-shock streams and sheath regions caused the largest fraction of intense magnetic storms $\left(D_{s t}<-100 \mathrm{nT}\right)$ during 1997-2000. The evolution of a storm caused by a magnetic cloud has many differences when compared to a storm caused by a post-shock stream or by a sheath region. Post-shock streams and sheath regions are associated with irregular behaviour of solar wind variables and high dynamic pressure. These features result in strong and variable convection strength (essential for trapping ring current particles), preconditioning of the nightside plasma sheet source population and strong high-latitude activity. Due to high dynamic pressure the subsolar magnetopause is compressed at times beyond the geostationary orbit. Probably the compressed and dynamic state of the magnetosphere also increases the geoeffectivity of these structures. 
Our study demonstrates that, on the other hand, sheath regions and post-shock streams may cause relatively intense and long-time disturbances at the high-latitude magnetosphere, while they have little or no effect at the low-latitude magnetosphere (i.e. decrease of $D_{s t}$ or $S Y M H$ ). These kind of events would be classified as large magnetic storms if the $K_{p}$ index only were used as a storm indicator. For the events presented here high-latitude activity was not followed by low-latitude activity until several hours later. Thus, having a global idea of the magnetospheric effects using different magnetic indices is necessary. It was shown that the magnetic activity generated by post-shock streams and sheath regions is more difficult to model probably due to more irregular solar wind conditions and the preconditioning of the nightside plasma sheet source population. To distinguish the general trend in the characteristic of magnetospheric activity generated by different solar wind drivers is helpful for space weather forecasting and for better understanding of magnetospheric dynamics.

Acknowledgements. The used magnetic indices $\left(K_{p}, A E, A L\right.$, $S Y M H, A S Y H$ and $D_{s t}$ ) were obtained from the World Data Center $\mathrm{C} 2$ in Kyoto. We thank R. Lepping for the WIND magnetic field data, and A. Lazarus for the WIND solar wind data. We also thank C. W. Smith for the ACE magnetic field data and, D. J. McComas for the ACE solar wind data. These data were obtained through Coordinated Data Analysis Web (CDAWeb). We want to acknowledge E. Tanskanen for comments on the substorm activity during storms. The study was supported through the Antares programme of the Academy of Finland.

The editor in chief thanks P.-O'Brien for his help in evaluating this paper.

\section{References}

Borovsky, J. E., Thomsen, M. F., and McComas, D. J.: The superdense plasma sheet: Plasmapheric origin, solar wind origin, or ionospheric origin?, J. Geophys. Res., 102, 22 089, 1997.

Burlaga, L., Sittler, E., Mariani, F., and Schwenn, R., Magnetic loop behind an interplanetary shock: Voyager, Helios and IMP 8 observations, J. Geophys. Res., 86, 6673-6684, 1981.

Burton, R. K., McPherron, R. L., and Russell, C. T.: An empirical relationship between interplanetary conditions and Dst, J. Geophys. Res., 80, 4204, 1975.

Daglis, I. A., Livi, S., Sarris, E. T., and Wilken, B.: Energy density of ionospheric and solar wind origin ions in the near-Earth magentotail during substorms, J. Geophys. Res., 99, 5691-5703, 1994.

Fenrich, F. R. and Luhmann, J. G.: Geomagnetic response to magnetic clouds of different polarity, Geophys. Res. Lett., 25, 29993003, 1998.

Ganushkina, N. Y., Pulkkinen, T. I., Kubyshkina, M. V., Singer, H. J., Russel, C. T., Reeves, G. T.: Long-term evolution of magnetospheric current during storms, Ann. Geophys., accepted, 2003.

Gonzalez, W. D., Joselyn, J. A., Kamide, Y., Kroehl, H. W., Rostoker, G., Tsurutani, B. T., and Vasyliunas, V. M.: What is a geomagnetic strom, J. Geophys. Res., 99, 5771-5792, 1994.

Gosling, J. T., McComas, D. J., Phillips, J. L., and Bame, J.: Activity associated with earth passage of interplanetary shock distur- bances and coronal mass ejections, J. Geophys. Res., 96, 78317839, 1991.

Greenspan, M. E. and Hamilton, D. C.: A test of the Dessler-ParkerSckopke relation during magnetic storms, J. Geophys. Res., 105, 5419-5430, 2000.

Huttunen, K. E. J., Koskinen, H. E. J., and Schwenn, R.: Variability of magnetospheric storms driven by different solar wind perturbations, J. Geophys. Res., 107(A7), 1-12, doi:10.1029/2001JA900171, 2002a.

Huttunen, K. E. J., Koskinen, H. E. J., Schwenn, R., and dal Lago, A.: Causes of major storms near the last solar maximum, in: Proceeding of The 10th European Solar Physics Meeting "Solar variability: From core to outer frontiers", edited by Wilson, A., 1 ESA Publication Division, ESTEC, 37-140, 2002b.

Huttunen, K. E. J., Koskinen, H. E. J., Pulkkinen, T. I., Pulkkinen, A., Palmroth, M., Reeves, E. G. D., and Singer, H. J.: April 2000 magnetic storm: Solar wind driver and magnetospheric response, 107(A12), 1440, doi:10.1029/2001JA009154, 2002c.

Iyemori T.: Storm-time magnetospheric currents inferred from midlatitude geomagnetic field variation, J. Geomag. Geoelectr., 42, 1249-1265, 1990.

Iyemori, T. and Rao, R. K.: Decay of the Dst field of geomagnetic disturbance after substorm onset and implication to stormsubstorm relation, Ann. Geophys., 14, 608-618, 1996.

Kamide, Y., McPherron, R. L., Gonzalez, W. D., Hamilton, D. C., Hudson, S., Joselyn J. A., Kahler, S. W., Lyons, R. L., Lundstedt, H., and Szuszczewicz, E.: Magnetic storms: Current understanding and outstanding questions, in: Magnetic Storms, edited by Tsurutani, B. T., Geophys. Monogr. 98, AGU, Washington D. C., 1-19, 1997.

Kozyra, J. U., Liehmohn, M. W., Clauer, C. R., Ridley, A. J., Thomsen, M. F., Borovsky, J. E., Roeder, J. L., Jordanova, V. K., and Gonzalez, W. D.: Multistep Dst development and ring current composition changes during the 4-6 June 1991 magnetic storm, J. Geophys. Res., 107(A8), doi:10.1029/2001JA000023, 2002.

Lepping, R. P., Berdichevsky, D. B., Burlaga, L. F., Lazarus, A. J., Kasper J., Desch, M. D., Wu, C. -C., Reames, D. V., Singer, H J., Smith, C. W., and Ackerson, K. L.: The Bastille Day magnetic cloud and upstrem shocks: near-Earth interplanetary observations, Solar Physics, 204, 287-305, 2001.

Liemohn M. W., Kozyra, J. U., Thomsen, M. F., Roeder, J. L., Lu, G., Borovsky, J. E., and Cayton, T. E.: Dominant role of the asymmetirc ring current in producing the stormtime Dst*, J. Geophys. Res., 106, 10 883-10 904, 2001.

Mayaud, P. N.: Derivation Meaning, and Use of Geomagnetic Indices, Geophys. Monogr. 22, AGU, Washington, D. C., 1980.

McPherron, R. L.: The role of substorms in the generation of magnetic storms, in Magnetic storms, edited by Tsurutani, B. T., 131-147, Geophys. Monogr. 98, AGU, Washington D. C., 1997.

O'Brien, T. P. and McPherron, R. L.: An empirical phase space analysis of ring current dynamics: Solar wind control of injection and decay, J. Geophys. Res., 105, 7707-7719, 2000a.

O'Brien, T. P. and McPherron, R. L.: Forecasting the ring current index Dst in real time, J. Atmos. S.-P., 62, 1295-1299, 2000 b.

Pulkkinen, T. I., Koskinen, H. E. J., Huttunen, K. E. J., Kauristie K., Tanskanen, E. I., Palmroth, M., and Reeves, G. D.: Effects of magnetic storms on substorm evolution, in: Proceedings of the 6th International Conference on Substorms, edited by Winglee, R. M., 464-471, 2002.

Richardson, I. G., Cliver, E. W., and Cane H. V.: Sources of geomagnetic storms for solar minimum and maximum conditions during 1972-2000, J. Geophys. Res., 28, 2569-2572, 2001. 
Sheeley, N. R., Howard, R. A., Koomen, M. J., Michelsand, D. J., Schwenn, R., Mahlhauser, K. H., and Rosenbauer, H.: Coronal mass ejections and interplanetary shocks, J. Geophys. Res., 90, $163-175,1985$.

Shue, J. -H., Song, P., Russel, C. T., Gosling, J. T., Steinberg, J. T., Chao, J. K., Zastenker, G., Vaisberg, O. L., Kokubun, S., Singer, H. J., Detman, T. R., and Kawano H.: Magnetopause location under extreme solar wind conditions, J. Geophys. Res., 103, 17 691-17 700, 1998.
Tsurutani, B. T., Gonzalez, W. T., Tang, F., Akasofu, S. I., and Smith, E.: Origin of interplanetary southward magnetic fields responsible for major magnetic storms near solar maximum (19781979), J. Geophys. Res., 93, 8519-8531, 1988.

Tsurutani, B. T. and Gonzalez, W. D.: The interplanetary causes of magnetic storms: A Rewiew, in: Magnetic Storms, edited by Tsurutani, B. T., 77-89, Geophys. Monogr. 98, AGU, Washington D. C., 1997.

Turner, N. E., Baker, N., Pulkkinen, T. I., and McPherron, R. L.: Evaluation of the tail current contribution to Dst, J. Geophys. Res., 105, 5431, 2000. 